\title{
La motivación en los negocios: estrategia para estimular la innovación en los diferentes tipos de negocios
}

\section{The motivation in business: strategy to stimulate innovation in different types of business}

\author{
Mariana, Bustamante \\ Universidad Estatal del Sur de Manabí, Ecuador \\ César, Bustamante \\ Universidad ECOTEC, Ecuador \\ Haydee, Yulan \\ Universidad Agraria del Ecuador, Ecuador \\ Zita, Zambrano \\ Universidad San Gregorio de Portoviejo, Ecuador
}

Autor para correspondencia: mariana.bustamante@unesum.edu.ec, cbustamante@ecotec.edu.ec Fecha de recepción: 23 de agosto de 2018 - Fecha de aceptación: 01 octubre de 2018

Resumen: Se presenta una experiencia en administración para la motivación en los negocios, que conduce a una idea de innovación, capaz de proporcionar una cultura empresarial en las diferentes ramas de los negocios. La misma toma en consideración relación entre las vivencias, experiencias y los saberes de los emprendedores que constituyen fuertes agentes dinámicos para la formación técnica, tecnológica, laboral y profesional. El estudio de la innovación destaca el papel de los negocios como agente innovador. Por esto el análisis buscará identificar no sólo la importancia de los negocios en la innovación, sino que se intentará establecer como la innovación se encuentra ligada a la orientación de los negocios. Las buenas ideas son inútiles a no ser que sean ejecutadas. La motivación del talento humano nace de situaciones opuestas así cuando las personas no están dispuestas al trabajo y huyen de el en cuanto pueden. Deben ser obligadas a trabajar, ser controladas y dirigidas. Por naturaleza son reacias al cambio. Por lo tanto, a las instancias administrativas en general, no les queda sino dos caminos: el duro de la coerción y amenaza, o supuestamente "dejar hacer" lo que a la gente plazca en base a la filosofía de la zanahoria y el garrote. He allí, palabras más o palabras menos, el meollo de la TEORIA "X" impulsada por la vieja escuela de administración del talento humano, misma que ha constituido el fundamento de acciones emprendidas por muchas empresas y gobiernos alrededor del globo. Este artículo está inmerso en los componentes de una buena estrategia de innovación.

Palabras Claves: motivación laboral; estrategia de innovación; educación profesional; administración; resultado artículo de innovación

Abstract: It presents an experience in management for business motivation, which leads to an idea of
innovation, capable of providing a business culture in different branches of business. It takes into
account the relationship between experiences, experiences and the knowledge of entrepreneurs who
are strong dynamic agents for technical, technological, labor and professional training. The study of
innovation highlights the role of business as an innovative agent. Therefore, the analysis will seek to
identify not only the importance of business in innovation, but also to try to establish how innovation
is linked to the orientation of business. Good ideas are useless unless they are executed. The motivation 
of human talent is born of opposite situations when people are not willing to work and flee from it as soon as they can. They must be forced to work, be controlled and directed. By nature they are reluctant to change. Therefore, to the administrative instances in general, they have only two ways: the hard of coercion and threat, or supposedly "letting do" what people like based on the philosophy of the carrot and the club. There, words more or less words, the core of the Theory "X" driven by the old school of human talent management, which has been the foundation of actions undertaken by many companies and governments around the globe. This article is immersed in the components of a good innovation strategy.

Key Words: work motivation; innovation strategy; professional education; administration; result innovation article

\section{Introducción}

La innovación se establece a partir de la importancia que permite a los negocios a conquistar nuevos mercados, aumentar la productividad, y mejorar la competitividad. Hoy día, ser competitivo sólo es posible de una manera sostenible con una alta productividad, ya que diferencias en coste de capital, materias primas, energía y transporte ya no son decisivos, es por ello que, el punto central alrededor del cual gira el presente trabajo es el estudio de la motivación en los negocios como ventaja competitiva en un contexto social de los negocios de la cámara de comercio del cantón Jipijapa. Además, de estudiar estrategias para estimular la innovación en los diferentes tipos de negocios como piedra angular del mejoramiento continuo de estas organizaciones, también se consideró relevante estudiar la importancia de la gestión de recursos humanos en el avance de la tecnología, causa que lleva a las organizaciones a cambiar su cultura mediante recursos de gestión para la competitividad, el objetivo fundamental fue: "Determinar la eficiencia en la motivación de los negocios mediante recursos de gestión para la competitividad de las empresas de la cámara de comercio del cantón Jipijapa, en la solución de problemas y en el análisis del objeto de estudio de las organizaciones, en el proceso administrativo.

El presente trabajo está estructurado de la siguiente manera. Primero se presenta una revisión de la literatura. A continuación, se explica la metodología del estudio. Luego se presentan y discuten los resultados. Y finalmente se presentan las conclusiones.

\section{Revisión de la literatura}

\section{Presupuestos básicos para estimular la innovación en los diferentes tipos de negocios}

Hay una relación positiva entre indicadores de la innovación y la productividad. Por tanto, resulta más fácil demostrar que una innovación deficiente origina una baja productividad y una pérdida del valor económico. Es fundamental evitar las consecuencias secundarias de una innovación deficiente, como son una espiral negativa de desmotivación, baja productividad, pesimismo, depresión, etc. La innovación introduce optimismo e ilusión en la compañía, logra la alineación de objetivos y forma equipos eficaces.

Si se analiza el término motivación observamos que se refiere a un viejo problema de la conducta, aunque es una categoría psicológica. Ella expresa lo relacionado con factores determinantes del comportamiento o con su causalidad: ¿Por qué laboramos?, ¿Por qué estamos activos o inactivos? De acuerdo con la concepción que tengamos, se entenderá el proceso 
económico, de ahí el papel importantísimo que desempeña en la optimización de los resultados, en la motivación) según (González, 2004)

A nivel de nuestro país el Ecuador se escucha quejas sobre el carácter parcial y limitado con el que la innovación ha sido entendida y tratada. Este Carácter que, por otro lado, simplemente trata de sintetizar otras limitaciones (por otra parte, evidentes) y obstáculos que dificultan el grado de innovación de un país. Estas limitaciones son ciertas, pero lo impostergable es no cejar en la búsqueda de la superación de estas realidades, para lo cual, como señala Seeley, "hay que construir sobre lo existente." (Seeley, 2001). En definitiva, quizás exista un camino que, como propone Bello, convierta "magros resultados en innovaciones radicales (...) innovar audaz y consistentemente, pero barato.” (Bello \& Gómez, 1996)

Opuesto al enfoque de la teoría "X", surgió la Teoría "Y", considerada como la moderna perspectiva sobre el trabajo y la administración. Sostiene que el trabajo es tan natural como el juego o el descanso. Postula que el ser humano busca tener más responsabilidades pues está cargado de iniciativas positivas y siempre está dispuesto a comprometerse con los objetivos de la organización, por lo que la administración puede minimizar las tareas de supervisión. Esta teoría es el justificativo de la moderna perspectiva sobre el trabajo y la administración, impulsada así mismo, por otros agentes sociales. Según (Paredes, 2011)

El aporte de estas teorías motivacionales corresponde a Douglas McGregor, teórico del comportamiento humano, quién las formuló en base a observaciones múltiples de la realidad laboral de su tiempo y sirvieron como explicación de conductas administrativas de distinto cuño.

A más de ellas y a manera de síntesis operativa, surgió la TEORIA “ $Z$ ” del investigador japonés Ouchi, quien sostuvo que la excelencia en el manejo administrativo pasaba, entre otras medidas, por garantizar la seguridad en el empleo a largo plazo, toma de decisiones colectiva, responsabilidad individual, y control informal e implícito, aunado a sistemas de medición del desempeño formales y explícitos. Propugnó además planes de vida y carrera moderadamente especializadas y la necesidad de interesarse en forma integral por el trabajador, incluyendo a su familia.

En el caso de nuestro país, ejemplos hay de aplicación a través del tiempo de las teorías mencionadas y la consiguiente repercusión en el convivir y el ambiente social considerado como un todo. ¿Cuál de estas tres teorías prevalece al momento? ¿Somos los ecuatorianos y ecuatorianas intrínsecamente buenos/as, o acaso nos gusta ser llevados del "mal modo"? ¿Qué porcentaje de la población tiene al momento el privilegio de un tratamiento como propugna la TEORIA “Z”? He allí temas de reflexión en que deberíamos participar activamente a fin de extraer conclusiones que contribuyan de manera efectiva al progreso colectivo.

Las teorías de la motivación en Psicología han sido de gran interés para el conocimiento humano.

Estudiar la motivación se han producido diversos enfoques que abarcan desde las teorías de la motivación humana, teorías de la motivación en el trabajo, teorías de la motivación del aprendizaje hasta teorías de la motivación más específicas. 
Maslow afirmó: "Un músico debe hacer música, un artista debe pintar, un poeta debe escribir, si quiere finalmente sentirse bien consigo mismo. Lo que un hombre puede ser, debe ser".Con ello, Maslow da a entender que no todos los hombres estarán motivados a satisfacer las mismas necesidades, por lo que está en cada uno satisfacer las necesidades que crea oportunas para llegar a su propósito.

\section{Estrategia para desarrollar la innovación en los diferentes tipos de negocios}

Según el artículo de los autores Wouter Koetzier, director global de gestión de la innovación y de la práctica de desarrollo de productos de Accenture, una compañía global de consultoría, tecnología y outsourcing, y Christopher Schorling, director general de Innovación Europea de Accenture y la práctica de desarrollo de productos de Accenture, creen que se debe fomentar un enfoque muy pragmático y orientado a la ejecución.

La estrategia de innovación define el papel de la innovación y establece la dirección que tomará la ejecución de la innovación. Sin embargo, el papel de innovación en ayudar a las organizaciones a alcanzar la meta del crecimiento es a menudo un poco claro y el crecimiento de los ingresos provenientes de la innovación no es suficiente a no ser que sean manejados con gran rigor. Si bien hay un montón de teorías y muchos buenos (Y no tan buenos) libros sobre la estrategia de innovación, las empresas no llegan a desarrollar y ejecutar una estrategia de innovación. Trabajamos con nuestros clientes para llegar a tener un enfoque más pragmático y orientado a la ejecución.

Una vez que se empieza a trabajar con un cliente para desarrollar una estrategia de innovación se busca desarrollar un entendimiento común de la definición del propósito de la estrategia de innovación. Cuando se habla de innovación en Accentures, se habla de la comercialización exitosa de nuevas ideas, es decir, invención con impacto en el mercado o en otras palabras, el concepto innovación = innovación x escalamiento. Sin embargo, la palabra nuevo puede tener diferente significado, que van desde nuevo en el mercado mundial a nuevo en una industria en específico, pero ya establecido en otro sector, a lo nuevo para una empresa o incluso solo nuevo para alguno de nosotros.

La palabra estrategia implica que estamos hablando de algo como un impacto potencialmente grande en la empresa, es decir no se refiere a una serie de mejoras incrementales a las líneas de productos.

Para Herbert Simon, el premio Nobel del Departamento de Sicología en la Universidad Carnagie-Mellon describía el reconocimiento de ideas como un proceso creativo que no es simplemente lógico, lineal y aditivo, sino que con frecuencia, el proceso es intuitivo, requiere una vinculación creativa o una asociación transversal, de dos o más "porciones" de experiencias, conocimientos técnicos y de contactos" (Timmons, 1999)

La innovación en los negocios se manifiesta en la forma en que es gestionada, aunque en el fondo este tipo de innovación no deja de ser una forma particular de innovación de procesos. 
Con este tipo de innovación se tiende a favorecer la capacidad de coordinación con el fin de que los negocios consigan responder mejor y más rápido a las exigencias de los clientes. Se trata de un tipo de innovación que puede cambiar el modelo de negocio, de un nuevo enfoque en la forma de satisfacer las demandas de los clientes que puede conllevar una nueva forma organizacional y que supone una nueva forma de competir en el mercado. Puede originarse tanto por la transformación de una empresa ya existente como por la aparición de una nueva empresa.

Entre las distintas estrategias de aplicación de la innovación en el sistema de gestión, se pueden destacar las siguientes por su mayor aplicación en la actualidad.

Según (Abella, 2002) El principal beneficio de la innovación empresarial se encuentra en la mejora de la calidad de los productos, sin obviar la importancia del aumento de la capacidad productiva y de la variedad de productos.

Algunos efectos posibles de la innovación empresarial son los siguientes:

- Aumento de la variedad de bienes y servicios.

- Aumento de la cuota de mercado.

- Mejora de la calidad de los bienes y servicios.

- Mejora en la flexibilidad productiva.

- Aumento de la capacidad productiva.

- Reducción de los costes laborales.

- Reducción del gasto de energía por unidad de producto.

- Mejora del medio ambiente.

Según (Internacional, 1998) En este sentido, la innovación empresarial puede reportar una serie de efectos concretos positivos para los negocios.

Según (Cooper R., 1999) una de las ventajas destacables es que puede suponer un ahorro en costes debido a la disminución de los procesos de fabricación o su optimización, un aumento de la productividad al mejorar los procesos de mecanización y disminuir la materia prima, así como un ahorro en el coste de la manipulación y el trasporte, al optimizar el espacio.

Otro de los beneficios de la innovación empresarial consiste en que puede afectar positivamente al proceso de distribución y venta, al mejorar el nivel de exposición del producto ante el consumidor, el acceso al producto, la colocación en los lineales, etc.

Un tercer beneficio a tener en cuenta son las posibles mejoras que la innovación empresarial puede inducir en la logística. Así, la introducción de la innovación puede ocasionar mejoras en cuanto a la distribución espacial de los almacenes o incluso la optimización de las cargas.

Por otra parte, según (Chan Kim, 1997) la innovación también puede incidir en una mejora de la información aportada al consumidor. De esta forma se puede conseguir una mejora en la visualización de los productos, facilitar una información más clara de las ventajas o prestaciones de los productos o incluso perfeccionar el reclamo en los puntos de venta mediante mensajes publicitarios impresos en los embalajes. 
La innovación también puede ser útil para realizar mejoras estéticas en los productos o servicios. A través del perfeccionamiento del envase o del rediseño de la imagen externa del producto, se pueden alcanzar presentaciones más atractivas para los clientes.

Desde el punto de vista medioambiental, otro efecto positivo de la innovación empresarial podría radicar en la disminución del peso y volumen del embalaje, con lo que se consigue una disminución de los residuos. En este sentido, también cabe destacar la posibilidad de reducir o eliminar los componentes contaminantes a través de la innovación empleada para utilizar materiales biodegradables.

En cuanto a la ergonomía del producto, uno de los posibles efectos de la innovación consiste en la mejora de los productos para conseguir una mayor facilidad de montaje, desmontaje y manejo.

Por otro lado, también se pueden conseguir mejoras a través de la innovación respecto al material consiguiendo progresos en cuanto a su ligereza, su limpieza, su estética o su precio.

Se debe tener en cuenta que uno de los efectos de la ausencia de innovación, cuando ya se han llevado a cabo acciones innovadoras, consiste en caer en la denominada "trampa de la explotación". Esto es, en determinadas ocasiones las empresas que ya han llevado a cabo alguna acción innovadora, por este hecho, dejan de vigilar el entorno y en consecuencia de reaccionar a sus posibles cambios.

Los efectos económicos de la innovación, ya que resulta vital para el crecimiento, no ya sólo para una empresa en particular, sino para la actividad económica a largo plazo de todo un país, en general. En efecto, la innovación es fuente de productividad y competitividad para las empresas, permitiendo consecuentemente una disminución de los precios de los bienes y servicios finales.

Según (FUNIVCYL, 2012) las innovaciones realizadas en un determinado sector productivo actúan recíproca e indirectamente en la productividad de otros sectores. De este modo, y a nivel empresarial, la innovación puede definirse como mejora de calidad y diferenciación del producto, lo que permite aumentar el valor añadido de las empresas.

El modelo SGS, se caracteriza, por una serie de inputs, criterios de salida, y un output. Los output típicos son decisiones de "siga/liquide/coseche/recicle". Cada puerta es administrada por un gerente ("portero"), siendo el conjunto de "porteros" multidisciplinario y multifuncional.

En cambio que para (Francisco, 1990) Tienen entre sus roles asegurar la calidad de los inputs, tomar decisiones sobre "siga... etc.", aprobar planes de acción para la etapa siguiente (si la decisión fue seguir) y decisiones sobre los recursos necesarios. Es elevado, entonces, el involucramiento gerencial, en particular de los gerentes senior; sin ellos, no el sistema no prospera. La tarea de estos líderes es, por tanto, compleja, multi-disciplinaria y multi-funcional.

Mantener los clientes existentes es siempre más rentable que adquirir otros nuevos, ya que consideramos que conseguir un nuevo cliente cuesta hasta 7 veces más que mantener uno existente. Y además, conservar los clientes habituales nos ayuda a predecir el flujo de trabajo y 
los posibles ingresos, a la vez que se hace más sencillo atenderlos debido al mayor conocimiento que vamos adquiriendo en el transcurso del tiempo.

Para (Zatarain, 1990) La relación de los temas tan recurrentes como: motivación, liderazgo, estrategias, estructura, desarrollo de subordinados (empowerment), entre otros, son temas de los especialistas del "management" en el fascinante mundo del "poder en las organizaciones"

Se reconocen dos principales fuentes de poder: "atributos personales" y la "posición que se ocupe". También se señala el papel de las estructuras, estrategias, y coaliciones (externas y externas) en los negocios.

La dependencia que exista entre los factores que se interrelacionen, influyen en el poder. A mayor nivel de dependencia, mayor poder tendrá el sujeto del que se dependa. Las alianzas externas e internas pueden ejercer un papel significativo en la utilización y distribución del poder en los negocios, en la medida en que pueden influir en estrategias y decisiones.

Se identifican las diferentes estrategias y tácticas que pueden aplicarse para ganar poder en los negocios.

Para (Wilson, 1979) las principales "estrategias de influencia" que se han identificado en los negocios con efectivos niveles de relaciones jefe-subordinados se señalan: la persuasión racional, apelación inspiracional, la consulta, comportamientos amistosos, intercambio (negociación), legitimización y presiones.

La calidad de vida laboral de una organización es el entorno, el ambiente, el aire que se respira en ella. Los esfuerzos para mejorar la vida laboral constituyen tareas sistemáticas que llevan a cabo los negocios para proporcionar a los empleados una oportunidad de mejorar sus puestos y su contribución a la empresa, es un ambiente de mayor confianza y respecto.

Motivación y satisfacción son dos elementos fundamentales en el ámbito de los negocios, necesidad de establecer nuevos contratos psicológicos para atraer y retener a nuevos empleados valiosos.

En la motivación debe depositarse la responsabilidad de la necesidad de encontrar formas de aumentar el rendimiento de los trabajadores con respecto a las tendencias negativas del desarrollo de los negocios.

La motivación laboral es una de la herramientas más útiles en los negocios, de allí el interés en ver de qué manera se puede estimular correctamente a los empleados, y el cómo el individúo está siendo manipulado por el ambiente laboral.

Conciliar la vida laboral y personal de los trabajadores en busca de una mejora del resultado empresarial. Viendo la oportunidad en lugar del inconveniente, hacer de esta ventaja competitiva, buscando la mejora de la productividad. 
Para (Nuttim J., 2014) una idea de negocio puede surgir por alguna de estas tres motivaciones

1. Por necesidad: las situaciones críticas estimulan la generación de ideas. Muchas personas se deciden a emprender cuando viven situaciones extremas, como puede ser el desempleo. Sin embargo en el mundo oriental, todas las crisis son una fuente de oportunidad y Según (Einstein, 1935) señalaba que "es en la crisis que nace la inventiva, los descubrimientos y las grandes estrategias. Quien supera la crisis, se supera a sí mismo sin quedar "superado".

2. Por vocación: son personas cuya vocación para ser emprendedores siempre ha estado latente y se gatilla por cualquier evento. Cuando niños, son los que venden limonadas, los que salen a trabajar en el verano, McClellan diría que tienen una alta motivación de logro y les vaticina muchos emprendimientos a lo largo de su vida. Tienen por lo tanto una postura proactiva para la generación de ideas y búsqueda de oportunidades, de cualquier tipo.

3. Por iniciativa: aquí se encuentran las personas que emprenden con la certeza que alcanzarán los resultados deseados, a diferencia del emprendedor por vocación, que "va a todas", el emprendedor por iniciativa, toma riesgos calculados frente a una oportunidad, se documenta y busca información y gestiona sus recursos para alcanzar sus objetivos y solamente emprende cuando los resultados serán mayores que su inversión.

Para (Ríos Martínez, 2011) Las ideas son consecuencia de ciertos factores, dentro de los cuales pueden destacarse: la observación del entorno, la formación, la experiencia, los gustos y los deseos del emprendedor. En la mayoría de los emprendimientos, fundamentalmente en los que tienen un componente de innovación, es fundamental que el emprendedor haga una adecuada búsqueda de información, que le permitirá captar información del exterior que podrá analizar y utilizar para generar nuevas ideas.

Es recomendable que las ideas tengan relación con la formación, la experiencia o el entorno del emprendedor. Una idea consiste en pensar las cosas diferentes, por ejemplo, considerar las ideas como solucionadores de problemas, también son una fuente de motivación en esta nueva era, los problemas medioambientales han dado origen a una serie de emprendedores y consumidores "verdes", con soluciones basadas en productos reciclados o fabricados con fibras naturales.

\section{Metodología}

La metodología utilizada fue la investigación cualitativa porque estudia la realidad en su contexto natural y como sucede, sacando e interpretando fenómenos de acuerdo con las personas implicadas. Utiliza variedad de instrumentos para recoger información como las entrevistas, imágenes, observaciones, historia de vida, en los que se describen las rutinas y la situación problemáticas, así como los significados en la vida de los participantes.

Por otra parte, (Taylor \& Bogdan, 1987) citados por Blasco y Pérez al referirse a la metodología cualitativa como un modo de encarar el mundo empírico, señalan que en su más amplio sentido es la investigación que produce datos descriptivos: las palabras de las personas, habladas o escritas y la conducta observable. 
Las técnicas y los instrumentos utilizados, se ciñeron a través de Técnicas de observación permitiendo hacer seguimiento del comportamiento de los representantes de la Cámara de comercio del cantón Jipijapa.

En las encuestas se emplearon cuestionarios dirigidos a representantes, empleados y autoridades de la Cámara de comercio del cantón Jipijapa, para saber si las actividades que realizan dichos empleados y autoridades han ayudado en la aplicación de las empresas y su rol fundamental en fomentar Estrategias para estimular la innovación en los diferentes tipos de negocios, y además conocer las actividades que tienen los empleados para la aplicación de las empresas en crear motivación en los negocios.

Análisis Y Discusión De Resultados

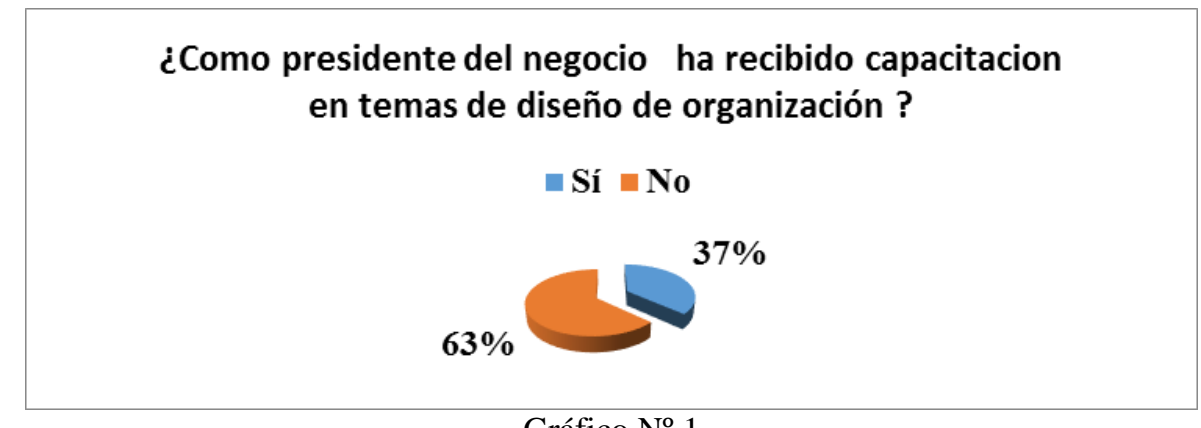

Gráfico $\mathrm{N}^{\circ} 1$

Fuente: Presidentes de negocios afiliados a la cámara de comercio del cantón Jipijapa.

Elaboración: propia

El análisis determina que $37 \%$ de los presidentes de negocios afiliados a la cámara de comercio del cantón Jipijapa han recibido capacitaciones en temas de diseño de organización perfecta que les permita obtener un rol fundamental en estrategias para estimular la innovación en los diferentes tipos de negocios y en su gran parte el $63 \%$ de los presidentes no han recibido estas capacitaciones, teniendo en cuenta que a pesar de recibir las capacitaciones muchos presidentes no han puesto en marcha sus conocimientos debido a la falta de tiempo .

A lo largo de las últimas décadas han sido varios los trabajos desarrollados con el fin de establecer cuál es el proceso que una organización ha de seguir en el desarrollo de un nuevo producto. Pese a que en este sentido las aportaciones de la literatura han sido amplias y variadas, se considera que de todos los trabajos presentados el más destacable es el desarrollado por Robert Cooper a lo largo de la década de 1990.

Frente a la evidencia del fracaso frecuente de desarrollo de nuevos productos -solamente uno de cada cuatro logra éxito- Robert Cooper propone una herramienta que a modo de solución, constituye una alternativa a la manía de las fusiones que se crean, en buena medida para evitar las experiencias frustrantes de desarrollo de negocios nuevos, sobre todo aquellos diversificados. Este instrumento es su notable sistema de "stage-gate system (puertas por etapa)", en el cual el desarrollo de un nuevo producto en definitiva es resultado de un doble proceso que se da en el tiempo de forma simultánea (Cooper, 1999) 


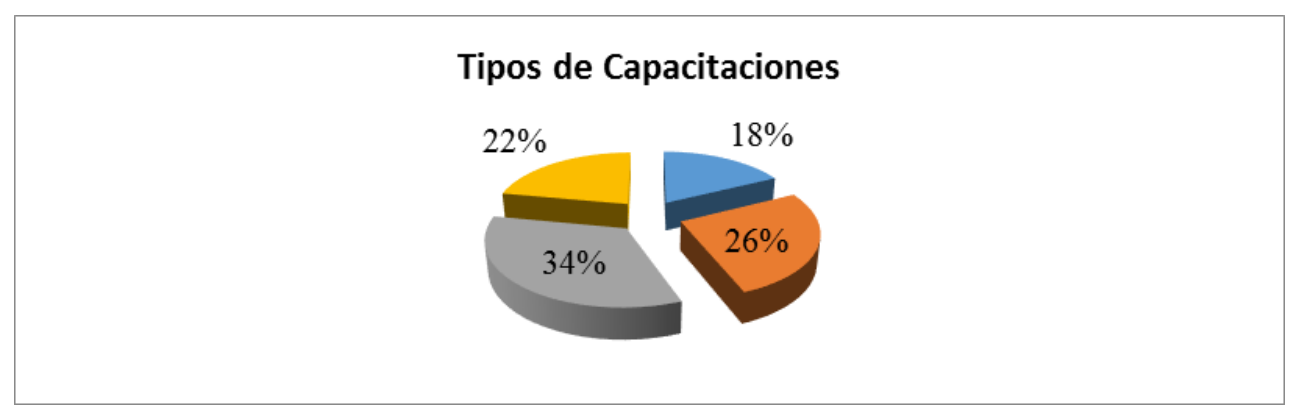

Gráfico $\mathrm{N}^{\circ} 2$

Fuente: Presidentes de negocios afiliados a la cámara de comercio del cantón Jipijapa

Elaboración: Propia

El análisis determina que mediante los resultados obtenidos el $18 \%$ de los presidentes de negocios miembros de la cámara de comercio del cantón Jipijapa que recibieron las capacitaciones fueron en base a temas de emprendimiento, el 26\% en temas de organización, un $34 \%$ en temas de finanzas y un $3 \%$ en temas adicionales. Cabe recalcar que los beneficiarios de estos temas de capacitación no han podido emprender por desconocimiento y desmotivación personal para lograr estos objetivos.

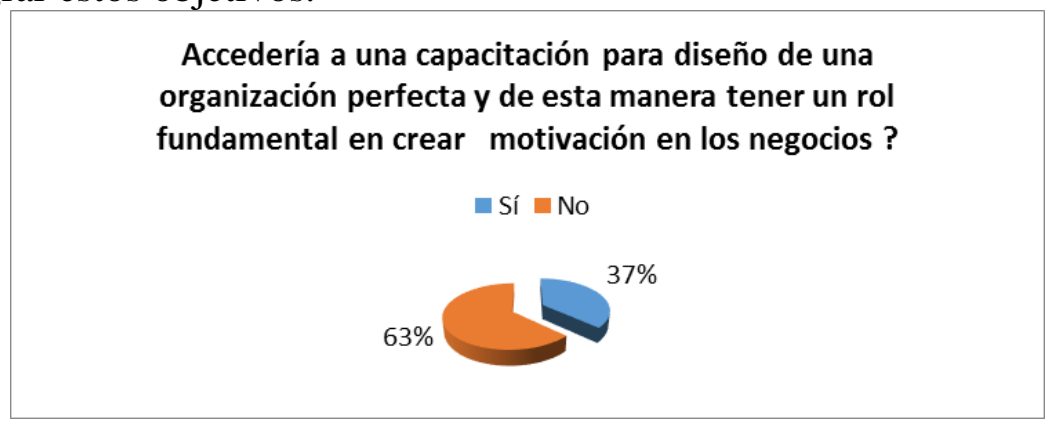

Gráfico $\mathrm{N}^{\circ} 3$

Fuente: Presidentes de negocios afiliados a la cámara de comercio del cantón Jipijapa Elaboración: Propia

El análisis determina que mediante los resultados obtenidos el 63\% de los Presidentes de negocios afiliados a la cámara de comercio del cantón Jipijapa no accederían a una capacitación para diseño de un negocio perfecta para empresas familiares y de esta manera tener un rol fundamental en crear motivación en los negocios, mientras que solo un $37 \%$ si desean acceder a una capacitación, ya que les ayudaría a conocer mejor este diseño organizacional.

Posteriormente a cada una de las fases, se desarrolla un proceso de control sobre la calidad del proceso, lo que es llamado puerta (gate). "Un conjunto de criterios de evaluación de la calidad es específico para cada puerta, de tal modo que si la evaluación es superada el proceso pasará a la siguiente fase." (Villegas, 1990)

\section{Conclusiones}

Las empresas son muy importantes para la economía de Ecuador. Sin embargo, la motivación y sus características hacen que éstas estén diferenciadas comparándolas con los que no poseen estrategias para estimular la innovación en los diferentes tipos de negocios. 
Ser competitivo sólo es posible de una manera sostenible con una alta productividad, ya que diferencias en coste de capital, materias primas, energía y transporte ya no son decisivos.

Esta realidad tiene ventajas y desventajas. Se podría decir que básicamente la ventaja es el fuerte compromiso de los integrantes de la cámara de comercio del cantón Jipijapa que motiven sus negocios aplicando estrategias para estimular la innovación.

Así, es imperativo compatibilizar los intereses de los distintos colectivos implicados en los negocios pertenecientes a la cámara de comercio del cantón Jipijapa, una gestión que favorece la existencia, a menudo latente, de tensiones en el seno de estos negocios.

En la presente investigación se realizó una caracterización de la motivación en los negocios, así como también estrategias para estimular la innovación; que permitió presentar un modelo de gestión para los negocios de la cámara de comercio del cantón Jipijapa.

\section{Bibliografía}

Abella, M. J. (2002). Encuesta Piloto de Actividades de Innovación en la industria. Uruguay: DINACYT (inédita).

Bello, L., \& Gómez, J. (1996). Las denominaciones de origen y otras señales de calidad en las estrategias de diferenciación. España: Cuadernos aragoneses de economía, $\mathrm{N}^{\circ}$ 6:2.

Cooper, R. (1999). The invisible success factors in product innovation. Journal of Producto Innovation Management, 16.

Cooper, R. (1999). The invisible success factors in product innovation. Journal of Producto Innovation Management,

Chan Kim, W. y. (1997). Innovación de valor: la lógica estratégica del alto crecimiento. En W. y. Chan Kim. Chile: Harvard Business Review.

Einstein, A. (1935). The World As I See It. Open Road Integrated Media, LLC. En A. Einstein.

Francisco, G. V. (1990). En G. V. Francisco, " Liderazgo ", Ed. Instituto de Capacitación Política. México D.F.: Ed. Instituto de Capacitación Política,

FUNIVCYL. (2012). Manual del Emprendedor Universitario. Castilla y León: Symbiosis Strategy \& Management Consulting, S.L.L. En FUNIVCYL.

González, L. A. (2004). La motivación hacia el estudio. Fundamentos y Metodología para su evaluación en Secundaria Básica. . Habana.

Internacional, C. (1998). Manual del Facilitador. Plataforma Aurea. En C. Internacional. Valparaíso, Chile. Nuttim, J. (2014). En Teoría de la motivación humana. Editorial Paidos.

Nuttim, J. (2014). Teoría de la motivación humana. En J. Nuttim. Editorial Paidos.

Paredes, E. (2011). Motivación del talento humano. En E. Paredes.

RÍOS MARTÍNEZ, W. y. (2011). En W. y. RÍOS MARTÍNEZ, Fortalecimiento De La Componente Creativa En La Formación Empresarial. . Universidad Icesi: Santiago de Cali.

Seeley, J. (Septiembre de 2001). El desafío de la innovación. . págs. Vol. 6, № 5.

Taylor, \& Bogdan. (1987).

Timmons, J. (1999). New Venture Creation Entrepreneurship for the 21st. Century. Boston: Irwin Mc Grew Hill.

Villegas, F. G. (1990). "Liderazgo ". México: Ed. Instituto de Capacitación Política.

Wilson, M. T. (1979). El ámbito de la motivación (ambiental. Fisiológica, mental y social). En M. T. Wilson. México: Editorial El Manual Moderno S.A.

Zatarain, I. M. (1990). “Redacción e Investigación Documental “, En I. M. Zatarain. UPN (Sep), México. 